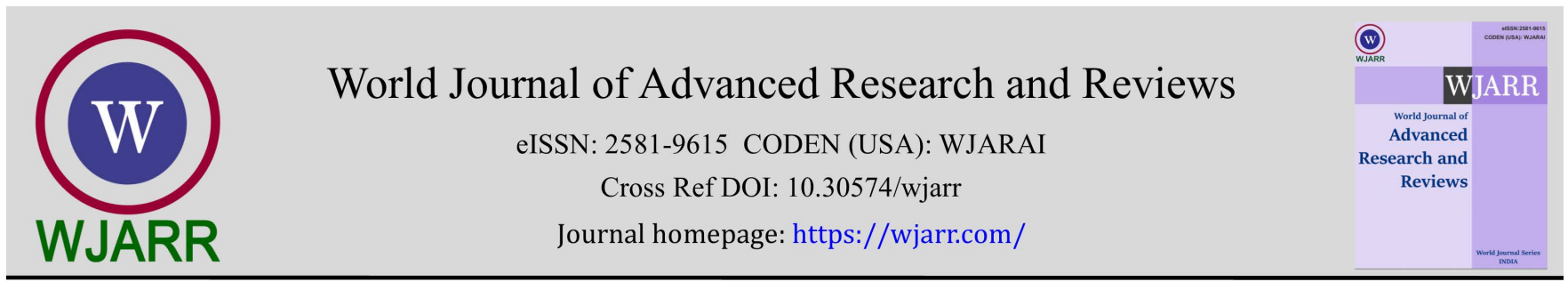

(RESEARCH ARTiCle)

Check for updates

\title{
Images Processing for Segmentation Neisseria Bacteria Cells
}

Kalthom Adam H. Ibrahim 1, ${ }^{*}$, Mohammed Abdallah Almaleeh 2, Moaawia Mohamed Ahmed ${ }^{2}$ and Dalia Mahmoud Adam 1

${ }^{1}$ Faculty of Engineering, Department of Electrical Control, El neelain University, Khartoum, Sudan.

${ }^{2}$ Faculty of Computer and Information Technology, Department of Computer Engineering, Tabuk University, Tabuk, KSA.

World Journal of Advanced Research and Reviews, 2021, 12(03), 573-579

Publication history: Received on 06 November 2021; revised on 18 December 2021; accepted on 20 December 2021

Article DOI: https://doi.org/10.30574/wjarr.2021.12.3.0672

\begin{abstract}
This paper introduces the segmentation of Neisseria bacterial meningitis images. Images segmentation is an operation of identifying the homogeneous location in a digital image. The basic idea behind segmentation called thresholding, which be classified as single thresholding and multiple thresholding. To perform images segmentation, transformations and morphological operations processes are used to segment the images, as well as image transformation an edge detecting, filling operation, design structure element, and arithmetic operations technique is used to implement images segmentation. The images segmentation represent significant step in extracting images features and diagnoses the disease by computer software applications.
\end{abstract}

Keywords: Diseases; Neisseria; Meningitis; Cerebrospinal fluid; Diagnose

\section{Introduction}

In computer vision techniques, segmentation is the process of partitioning a digital image into multiple segments sets of pixels, also known as super pixel[1]. The goal of segmentation is to simplify or change the representation of an image into something that is more meaningful and easier to analyze. Image segmentation is implemented in various applications, for all the applications, individual method cannot produce the desired result; it is due to that the images have different property and some other factors like noise, and intensity resolution[2].

Image fragmentation algorithms generally based on one of two basic properties of density values: discontinuity and similarity[3]. In the first class, the approach is to divide Image segmentation algorithms generally based on one of two basic properties of intensity values: discontinuity and similarity[4]. In the first category, the approach is to partition an image based on abrupt changes in intensity, an edge-detection algorithms fall in this category. In the second approach, an image partitioned into regions that are similar according to set of defined criteria[5]. Thresholding and watershed segmentation fall in this category. Many biological images contain of light objects over a constant dark background (especially those obtained using fluorescence microscopy); in such a way that object and background pixels have gray levels grouped into two dominant modes[6].

Diagnostic imaging is a vital tool in medicine nowadays. Magnetic resonance imaging (MRI), computed tomography (CT), digital mammography, and other imaging modalities provide an effective means for noninvasively mapping the anatomy of a subject[7]. These tools have greatly increased knowledge of normality and diseased anatomy for medical research and are essential component in diagnosis and planning, within increasing size and number of medical images, the use of computers in facilitating images treating and analysis has become an essential. In particular, computer algorithms for the outlining of functional arrangements and other areas of interest are a key component in supporting

\footnotetext{
${ }^{*}$ Corresponding author: Kalthom Adam H. Ibrahim

Department of Electrical Control, Faculty of Engineering, El neelain University, Khartoum, Sudan.

Copyright (C) 2021 Author(s) retain the copyright of this article. This article is published under the terms of the Creative Commons Attribution Liscense 4.0.
} 
and regulating specific radiological responsibilities[8]. This paper is about the application of image segmentation techniques by taking a digital light microscope image of a smear of Cerebrospinal fluid (CSF) that diagnosed in the laboratory by chemical methods as being infected with the Neisseria bacterium that causes meningitis.

\section{Related Work}

A great deal of research has been done in medical image diagnosis. The medical segment is essentially the cornerstone of medical image diagnosis using computer applications. Advances in imaging technologies such as computer tomography (CT) and magnetic resonance imaging (MRI) have made available to clinicians high-resolution images that have greatly aided in clinical diagnosis[9]. Segmentation is a reciprocal method so it is difficult to list most segmented areas. The key to segmentation is a digital grayscale medical image (CT or MRI). In this work, optical microscopy images are used to perform the segmentation process.

\section{Methodology}

In this work, samples were collected from cerebrospinal fluid from Al-Amin Hamid Children's Hospital in Omdurman, Sudan. The total number of cerebrospinal fluid smear images taken is 40 , which were affected by Neisseria bacteria, and the scale of the images is $300 \mu \mathrm{m}$. CSF blot images in red, green, and blue (RGB) colors are the product of the image acquisition stages; then the images are segmented. Matlab version Ra2014 was used to perform this work. Segmentation of the RGB smear image undergoes several operations. In these steps, bacteria cell boundaries are determined, and each bacterial cell is cropped from other surrounding CSF components into a sub-image containing only one bacteria cell on a white background. Techniques implemented are converting RGB image to binary images. Edge mode by Sobel and Prewitt methods, applying mathematical morphology such as filling process, and merging using structural design element (disk) to form a threshold of a reliable segmentation algorithm. This lead to extraction of images of bacterial cells, which is an area of interest, its internal component in a black and white image[10]. Figure 1 shows the image segmentation process. Bacterial images were isolated by cutting them from the rest of the image components in order to extract certain characteristics from the bacterial image and calculate the error resulting from the process of cutting bacteria images as an alternative to the computerized segmentation process.

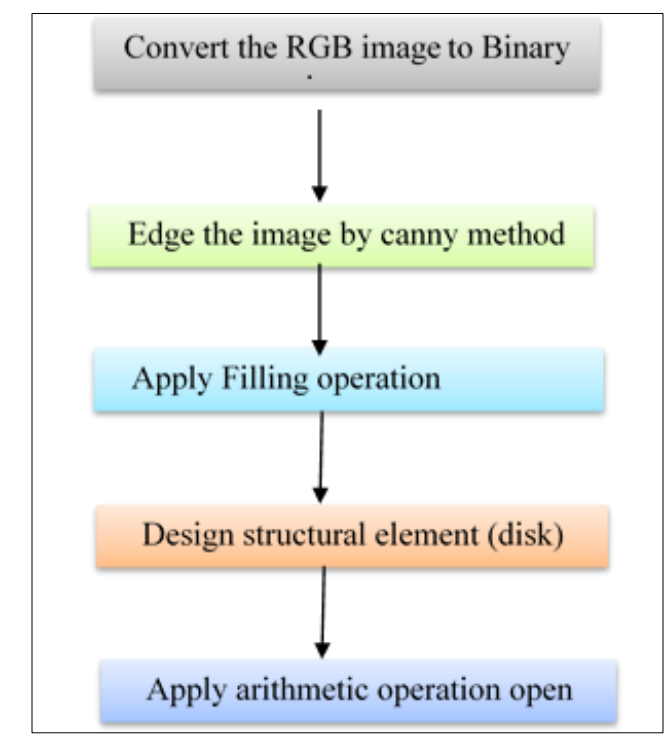

Figure 1 Images segmentation steps

\section{Results and discussion}

The result of image segmentation is a set of segments that cover the entire image, or a set of contours extracted from the image. Each of the pixels in a region is similar with respect to some characteristic[11]. There are no images enhancement done in this data acquisition such as noise reduction, stabilizing the color variation, illumination imbalance and lower uniform luminous intensity. Therefore, re-processing steps not needed to address the image flaws and to prepare the data for further analysis. The images in figure (2) to figure (6) shows the result of the CSF smear images segmentation algorithm. 
The segmentation images of CSF smear are the groundwork for the features extraction then the classification process of the images[12]. The result of image segmentation is a set of segments that collectively cover the entire image, or a set of contours extracted from the image. Each of the pixels in a region is similar with respect to some characteristic or computed property, such as color, intensity, or texture. Adjacent regions are significantly different with respect to the same characteristic[11].

In this work, an image segmentation based approach performed by using mathematical and morphology operation. In spite of the variation in the smear staining and the way of scanning the smear, however the CSF smear images colors appear in each bacteria image by the same or approximately same ranges. In the normal CSF smear images appear by another different way in color from the images infected by bacteria .Image segmentation technique depends on different methods that be followed to complete the process accurately for each group of images separately and each image has a different experience[13].

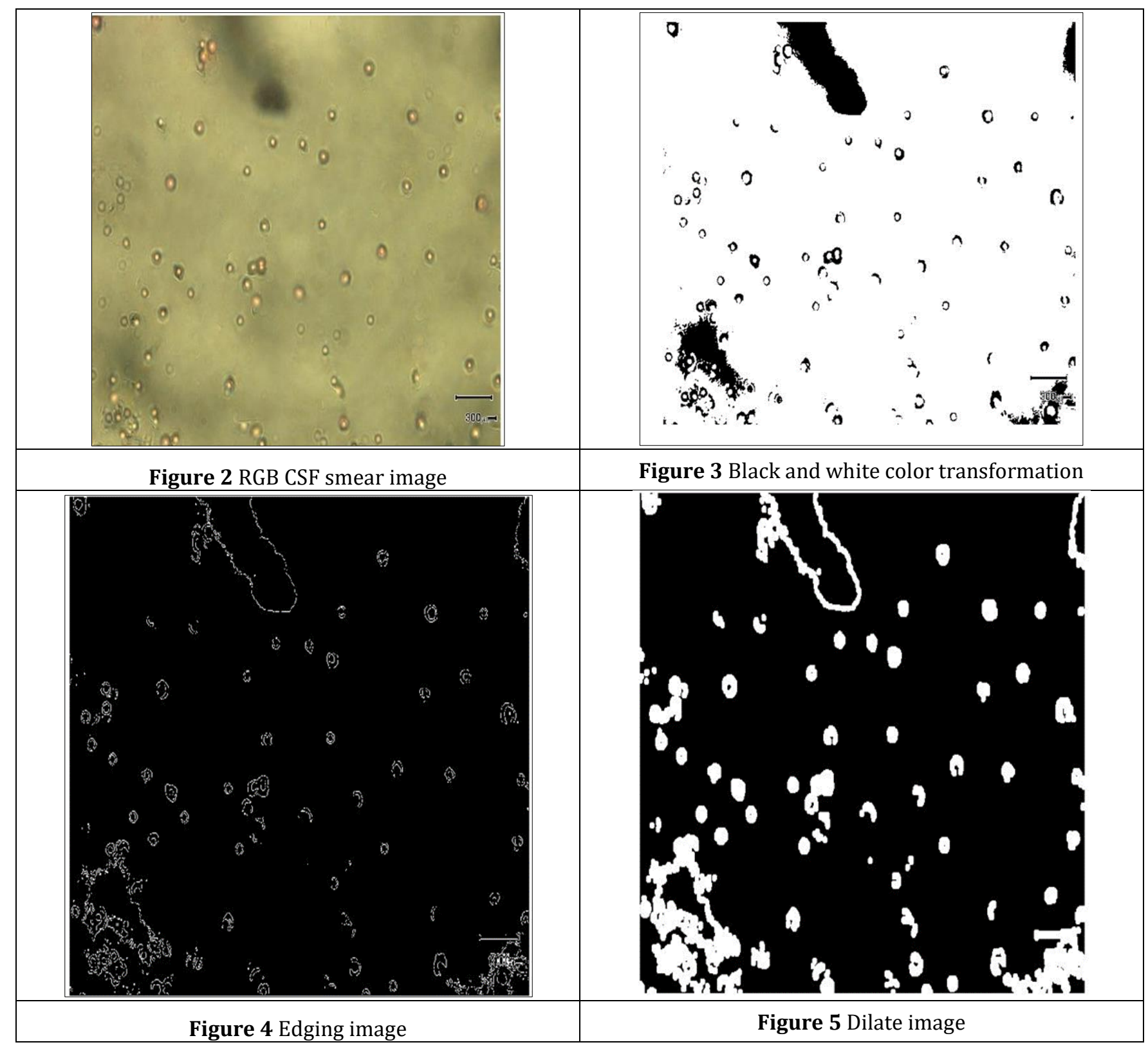




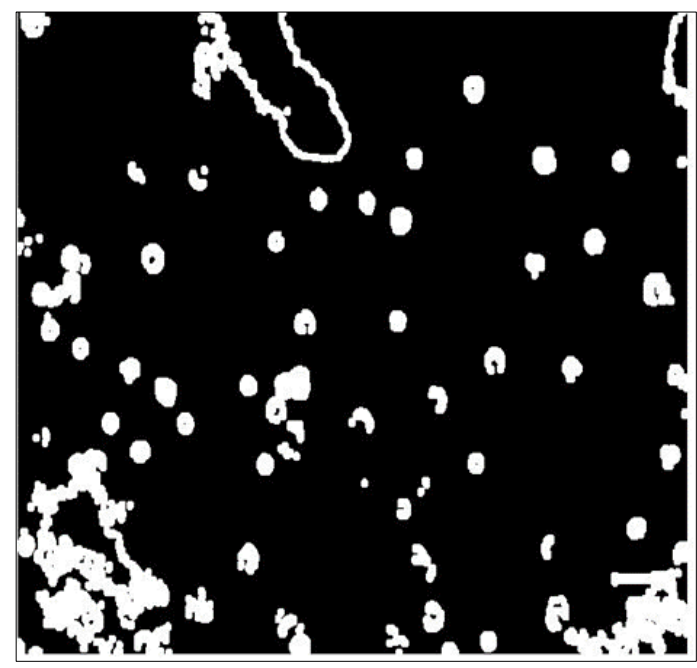

Figure 6 Segmented image

\section{Geometrical evaluation of the isolated bacteria images}

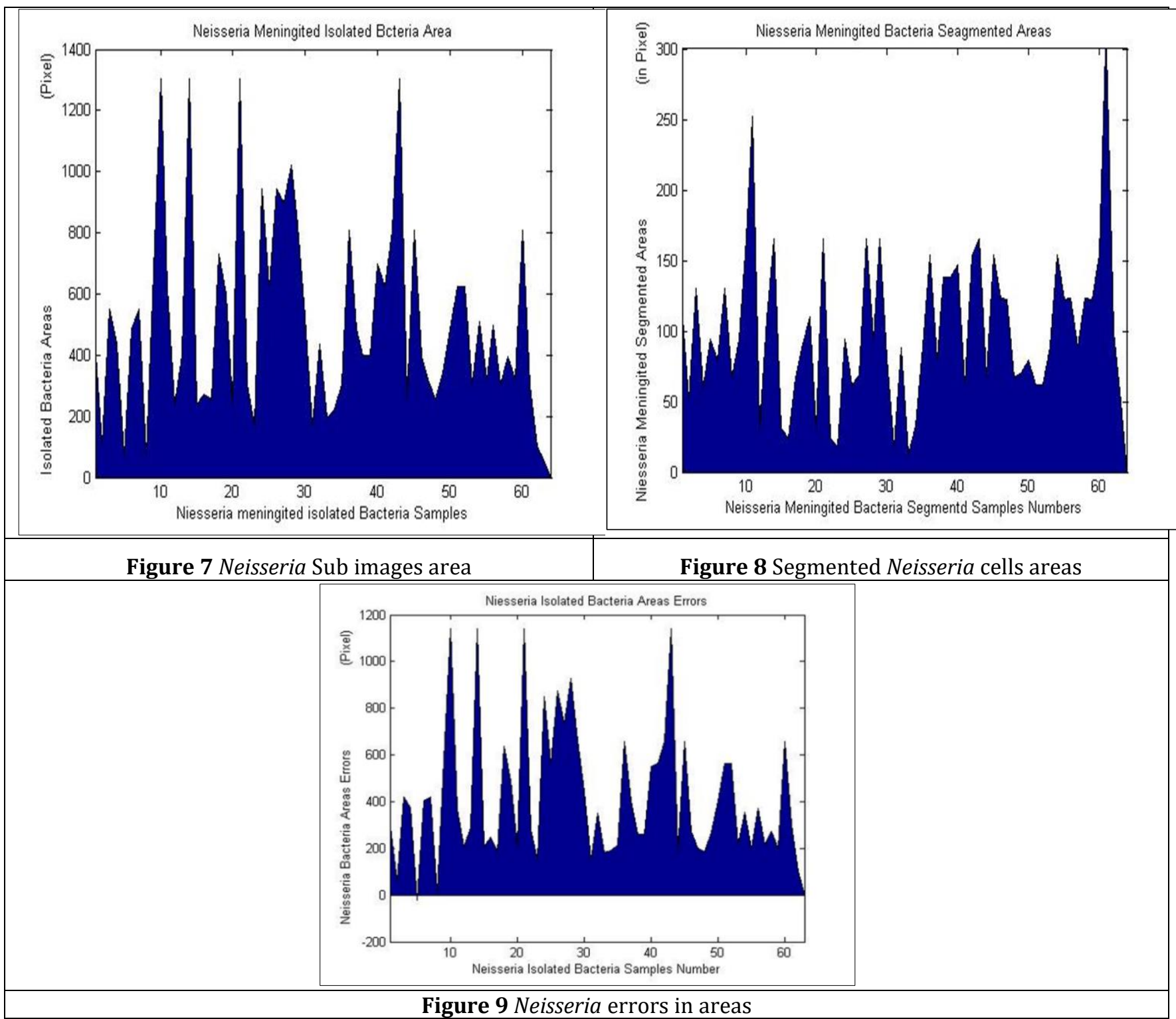


The errors in isolated bacteria images process evaluated by geometrical methods .Fig (7) illustrate the Neisseria sub bacteria images area fig (8) illustrate the Neisseria segmented areas, fig (9) illustrate the geometrical errors resulting from the variation in the areas of isolated bacteria's and segmented images for Neisseria. The mathematical formula, which used is the error in bacteria cell area=sub bacteria cell area-segmented bacteria cell area[14].

\section{Statistical evaluation of the isolated bacteria images}

The elemental mathematical linear and non-linear relations between measured values of bacteria cell area are essential in order to use linear regression for the calculation of the optimums of the desired values[15]. For the Neisseria types of bacteria, the linear requisition for the optimal values shown in fig (10). The linear regression is powerful tool for analyzing the data. To evaluate the regression errors for the isolated bacteria it is assumed that the values of the isolated Bactria areas is represented by the variable $\mathrm{X}$ and the values of segmented Bacteria is represented by $\mathrm{Y}$ variables, applying the linear regression errors equation below[16].

$$
\mathrm{Y}=\mathrm{a} \mathrm{X}+\mathrm{b}
$$

Where: $\mathrm{Y}$ is segmented Bacteria areas.

$\mathrm{X}$ is isolated Bactria areas

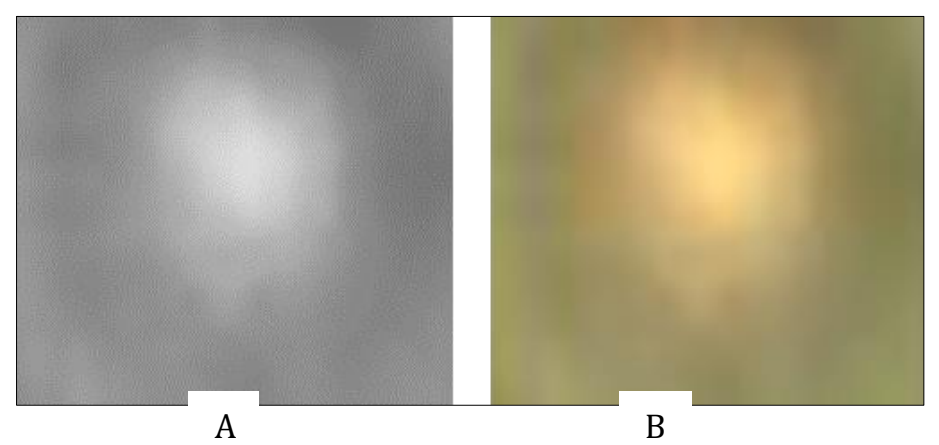

Figure 10 Isolated bacteria image. (a)Isolated Neisseria (gray color)

(b) Isolated Neisseria (colored)

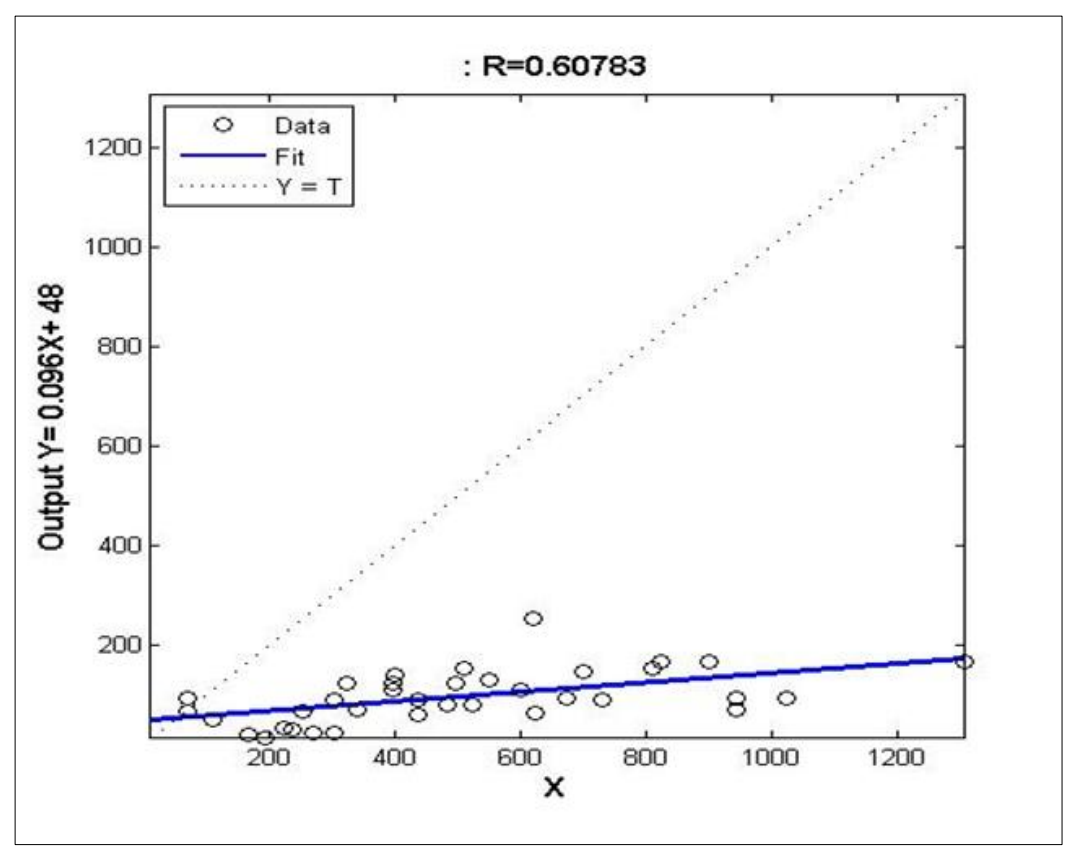

Figure 11 Neisseria isolate cell regression errors 


\section{Conclusion}

This is a segmentation algorithm designed to extract a single bacterial cell from the other CSF components shown in the image and separate the bacteria region from the CSF region. There are several ranges of image processing techniques are used for bacteria cell isolation, such as color level conversion image threshold, mathematical operations, and modulation[17]. A gradient edge detector designed and used to detect edges. In edge-based segmentation algorithms, Canny's algorithm produced the best hash compared to Sobel and Prewitt[18]. The proposed methodology achieved acceptable results and the bacteria region separated from the CSF region. In edge-based segmentation algorithms, But there are a variety of image segmentation techniques that can be applied to obtain a distinctive result for the bacterial classification process[19]. When the image is cut to isolate it from other image components, errors occur in the extracted image area, which results in errors when extracting image features to complete the automatic classification process[20].

\section{Compliance with ethical standards}

\section{Acknowledgments}

Authors are thankful for the research department of Khartoum state Health ministry and Al Amin Hamid children hospital, special thanks to laboratory staff for their continuous support in our research. The authors are grateful to Sara contactors and Al Adwa Alsara Company (Sharjah, U.A.E) for moral and material support. Special thanks expanded to those who contributed to the research with support, guidance, or care.

\section{Disclosure of conflict of interest}

There is no conflict of interest in this study.

\section{References}

[1] R. Achanta and S. Susstrunk, "Superpixels and polygons using simple non-iterative clustering," in Proceedings of the IEEE Conference on Computer Vision and Pattern Recognition, 2017, pp. 4651-4660.

[2] L. Wang, T. Tan, W. Hu, and H. Ning, "Automatic gait recognition based on statistical shape analysis," IEEE transactions on image processing, vol. 12, no. 9, pp. 1120-1131, 2003.

[3] N. M. Zaitoun and M. J. Aqel, "Survey on image segmentation techniques," Procedia Computer Science, vol. 65, pp. 797-806, 2015.

[4] Y. Ramadevi, T. Sridevi, B. Poornima, and B. Kalyani, "Segmentation and object recognition using edge detection techniques," AIRCC's International Journal of Computer Science and Information Technology, vol. 2, no. 6, pp. 153$161,2010$.

[5] P. D. R. Raju and G. Neelima, "Image segmentation by using histogram thresholding," International Journal of Computer Science Engineering and Technology, vol. 2, no. 1, pp. 776-779, 2012.

[6] S. Bolte and F. P. Cordelières, "A guided tour into subcellular colocalization analysis in light microscopy," Journal of microscopy, vol. 224, no. 3, pp. 213-232, 2006.

[7] H. Kekre, S. M. Gharge, and T. K. Sarode, "Image segmentation of MRI images using vector quantization techniques," in Thinkquest 2010: Springer, 2011, pp. 171-176.

[8] S. K. Bandyopadhyay, "A survey on brain image segmentation methods," Journal of Global Research in Computer Science, vol. 2, no. 2, 2011.

[9] K. Hegmann et al., "Cervical and thoracic spine disorders," Occupational medicine practice guidelines Evaluation and management of common health problems and functional recovery in workers. 3rd ed. Elk Grove Village, Illinois, USA: American College of Occupational and Environmental Medicine (ACOEM), pp. 1-332, 2011.

[10] V. R. Borges, M. C. F. de Oliveira, T. G. Silva, A. A. H. Vieira, and B. Hamann, "Region growing for segmenting green microalgae images," IEEE/ACM transactions on computational biology and bioinformatics, vol. 15, no. 1, pp. 257$270,2016$.

[11] K. K. Singh and A. Singh, "A study of image segmentation algorithms for different types of images," International Journal of Computer Science Issues (IJCSI), vol. 7, no. 5, p. 414, 2010. 
[12] S. S. Chouhan, A. Kaul, and U. P. Singh, "Image segmentation using computational intelligence techniques," Archives of Computational Methods in Engineering, vol. 26, no. 3, pp. 533-596, 2019.

[13] G. L. Voigt and S. L. Swist, Hematology techniques and concepts for veterinary technicians. John Wiley \& Sons, 2011.

[14] O. A. Peters, A. Laib, T. N. Göhring, and F. Barbakow, "Changes in root canal geometry after preparation assessed by high-resolution computed tomography," Journal of endodontics, vol. 27, no. 1, pp. 1-6, 2001.

[15] G. A. Seber and A. J. Lee, Linear regression analysis. John Wiley \& Sons, 2012.

[16] J. V. T. Lara, Testing Foundational Tenets of Stable Isotope Ecology Analyses in Neotropical Mammalian Communities, and Implications for Terrestrial Paleoecology. Columbia University, 2020.

[17] N. T. Huy, N. T. Thao, D. T. Diep, M. Kikuchi, J. Zamora, and K. Hirayama, "Cerebrospinal fluid lactate concentration to distinguish bacterial from aseptic meningitis: a systemic review and meta-analysis," Critical care, vol. 14, no. 6, pp. 1-15, 2010.

[18] B. Rashmi and H. Nagendraswamy, "Video shot boundary detection using block based cumulative approach," Multimedia Tools and Applications, vol. 80, no. 1, pp. 641-664, 2021.

[19] P. Mesejo, O. Ibáñez, O. Cordón, and S. Cagnoni, "A survey on image segmentation using metaheuristic-based deformable models: state of the art and critical analysis," Applied Soft Computing, vol. 44, pp. 1-29, 2016.

[20] D. T. Nguyen, T. D. Pham, N. R. Baek, and K. R. Park, "Combining deep and handcrafted image features for presentation attack detection in face recognition systems using visible-light camera sensors," Sensors, vol. 18, no. 3, p. 699, 2018.

\section{Authors short Biography}

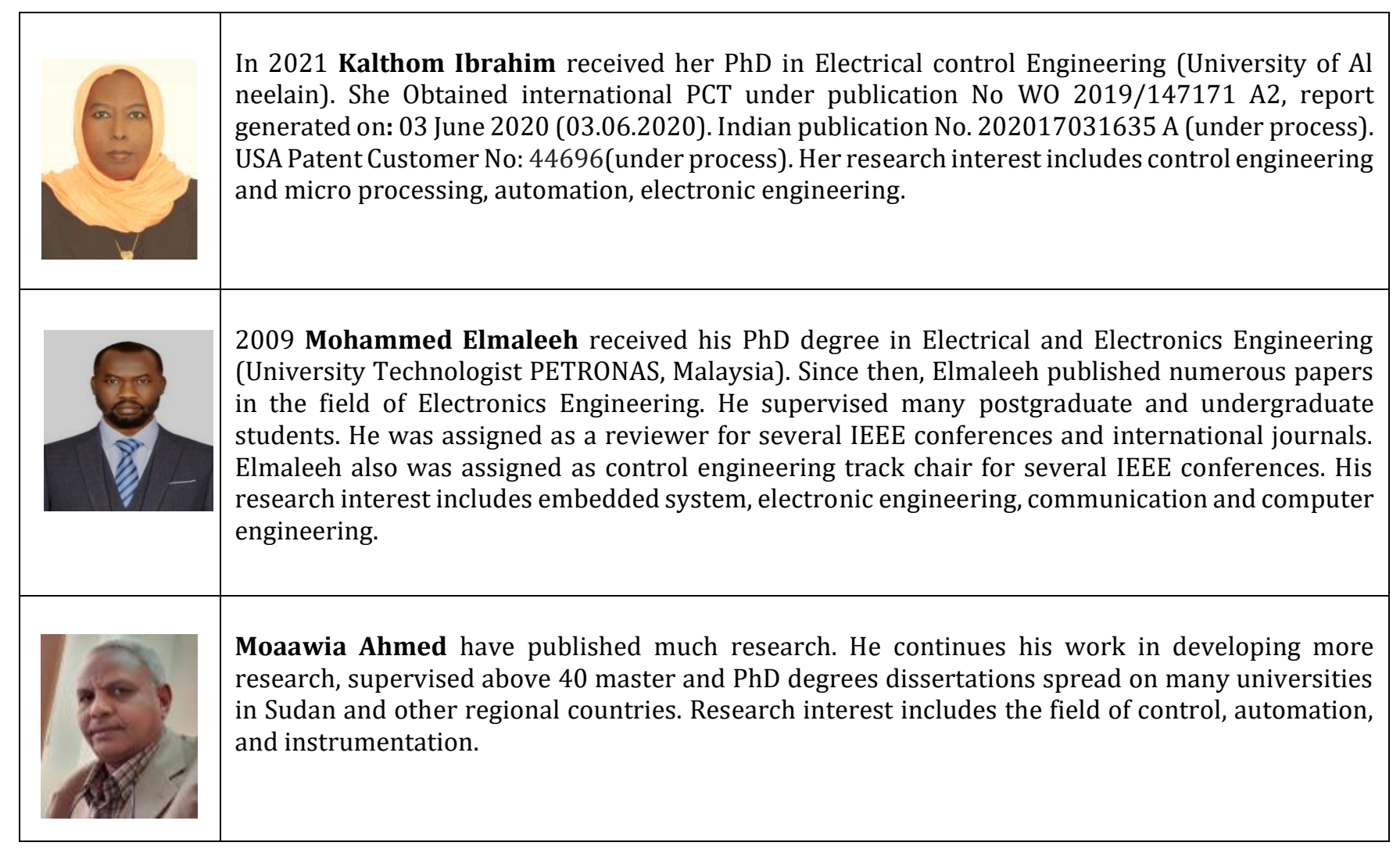

